\title{
Design and Analysis of Drag-bar of Planter Based on Modal Analysis
}

\author{
Yu Yang ${ }^{1, a}$ \\ ${ }^{1}$ Department of Communication Engineering Engineering College of Armed Police Force, Xi'an, \\ China \\ amiaoyude@163.com
}

Keywords: Drag -bar; ANSYS; Pro/E; modal analysis

Abstract. Three -dimensional model of drag-bar for 2BQ -2 planter was developed through Pro /E , the model was then transferred into ANSYS for analysis, and modal parameters of the drag-bar were calculated, natural frequency and vibration features of the drag-bar were determined. Modal frequency was concentrated in $0-1 \mathrm{~Hz}$ which was main vibration band and planting quality would be negatively affected when sympathetic vibration occurred as outside inspire frequency in accordance with natural vibration frequency of the drag -bar. The drag-bar would be in pendulum vibration when vibration frequency was at $0.23 \mathrm{~Hz}$. The results of the study provided reference for optimization design of the drag-bar of the planter.

\section{Introduction}

Planter drag-bar is an important part in the structure of planter. The deformation and failure of the drag-bar will affect the performance and safety of the planter. The traditional design of planter drag-bar is to use the analogy method and the strength check. It caused the strength of drag-bar is too large and materials waste[1].The dynamic design and analysis of the planter drag-bar with the modal analysis technique can get more accurate structure natural frequency, mode shape, modal damping, modal mass and modal stiffness. So the structure design can avoid the frequency or minimize the excitation of these frequencies, so as to eliminate the excessive vibration and ensure the reliability of the structure[2].

ANSYS Workbench finite element analysis makes use of element node variables to carry on interpolation for unit internal variables and achieve the overall structure of the analysis[3]. Using the modal analysis function of the finite element software, the finite element model of the planet drag-bar is established, and the natural frequency and vibration mode of the structure are determined, so as to describe the state of traction by dynamic image. This has important reference value for the design of drag-bar.

\section{Drag-bar model establishment}

Pro /E is used to model 2BQ-2 planter drag-bar produced by HeiShan mechanical manufacturing limited company and its 3D model is shown in Fig 1.

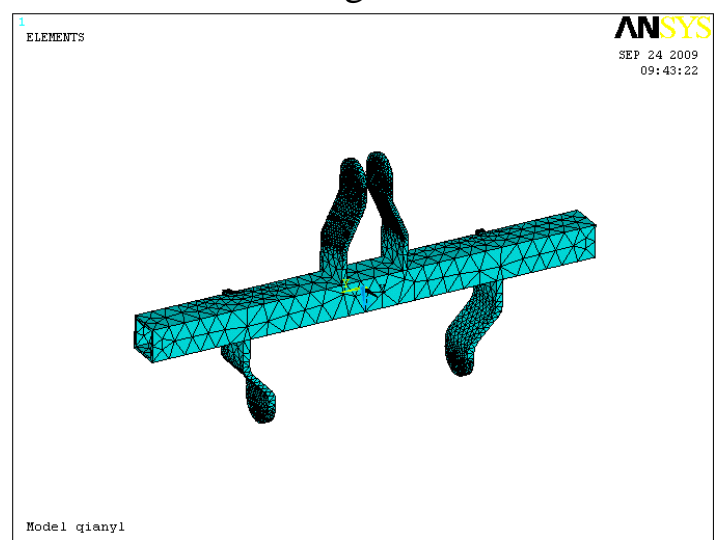

Fig. 1 Structure model of drag-bar 
Although the ANSYS software has the function of modeling, its function is not strong enough. So the ANSYS software is set up with a variety of data exchange interface of CAD software, such as Pro /E, UGNX4, AutoCAD. By ANSYS and interface setting of Pro/E, ANSYS is integrated directly into Pro/E, so as to realize the seamless connection of two kinds of software and ensure the accuracy of the Pro/E model into ANSYS. This method has very important significance to shorten the design time and speed up the design of the product.

\section{Drag-bar modal analysis}

Modal analysis theoretical Basis. The modal analysis of natural frequencies and mode shapes is the basis of other vibration analysis[4]. Through the modal analysis of the structure, designer can choose the reasonable structure design scheme, and verify the validity of the structure. The modal analysis of the planter drag-bar is an important part of its structure design, which is the premise of static test and dynamic analysis. Multi freedom system is presented in a certain natural frequency of vibration, and the vibration of the system is a certain proportion, which is called natural vibration mode[5]. In any case, the response of each point on the mechanical structure can be expressed as the superposition of the modal parameters of natural frequencies, damping ratio and mode shapes [6].The core content of modal analysis is to determine the parameters of the structure system dynamic characteristics.

For a $\mathrm{N}$ degree of freedom linear system, differential equations of motion is shown in formula (1)

$$
[M]\{\ddot{X}\}+[K][X]=\{0\}
$$

$[M]$ is mass matrix; $[K]$ is stiffness matrix; $\{\ddot{X}\}$ is acceleration vector; $\{X\}$ is displacement vector.

If the structure is vibrating at a fixed frequency and mode, it is shown in formula (2).

There is $\{\ddot{X}\}=-\omega^{2}\{\phi\} \sin (\omega t+\varphi)$

Into the equation of motion, free vibration characteristics of structural equation is shown in formula (4).

$$
\left([K]-\omega^{2}[M]\right)\{\phi]=\{0]
$$

$\{\phi\}=\{0\}$ is a solution. But this solution represents that all particles of the structure are in a static state. For the non zero solution, formula (5) must be satisfied.

$$
\operatorname{det}\left([K]-\omega^{2}[M]\right)=0
$$

formula (5) is called the eigenvalue equation of structure vibration. $\omega_{i}^{2}$ is eigenvalue of equation. It is back into the characteristic equation, the feature vector $\left\{\phi_{i}\right\}$ can be obtained. The square root of the characteristic value $\omega_{i}$ is the natural frequency of the structure. Feature vector $\left\{\phi_{i}\right\}$ is the shape vector structure corresponding to $\omega_{i}$.

Modal analysis. The main steps and procedures of modal analysis are shown in Fig 2. The 3D model of the drag-bar is introduced into ANSYS by Pro/E, and the finite element analysis is carried out. Specific steps are as follows:

1) Defining element type, select "Structural Solid Brick 20 node 95"

2) Defining material properties, material is 45 steel, modulus of elasticity is $205 \mathrm{GPa}$, Poisson ratio is 0.3 , density is $7820 \mathrm{~kg} / \mathrm{m} 3$.

3) Dividing cell, select "pick all" in "preprocessor eshing esh Tool"

4) By applying boundary and restriction condition, the drag-bar and the connecting hole of the connecting hole with three points are all bound;

5) Specify analysis type, select "Modal" in "Type of Analysis".

6) Specify analysis options, input 6 in "No. of modes to extract"

7) Solving, Select the OK option for Current Load Step Solve for its solution

When modal analysis is carried out, the natural frequencies and mode shapes are not required, but the frequency of the system under the operating conditions of the system is emphasized. Because the 
natural frequencies of these orders usually cause system resonance. The first 6 natural frequencies of the planter drag-bar are calculated and shown in Table 1, and the mode shapes are drawn and shown in Fig 3.

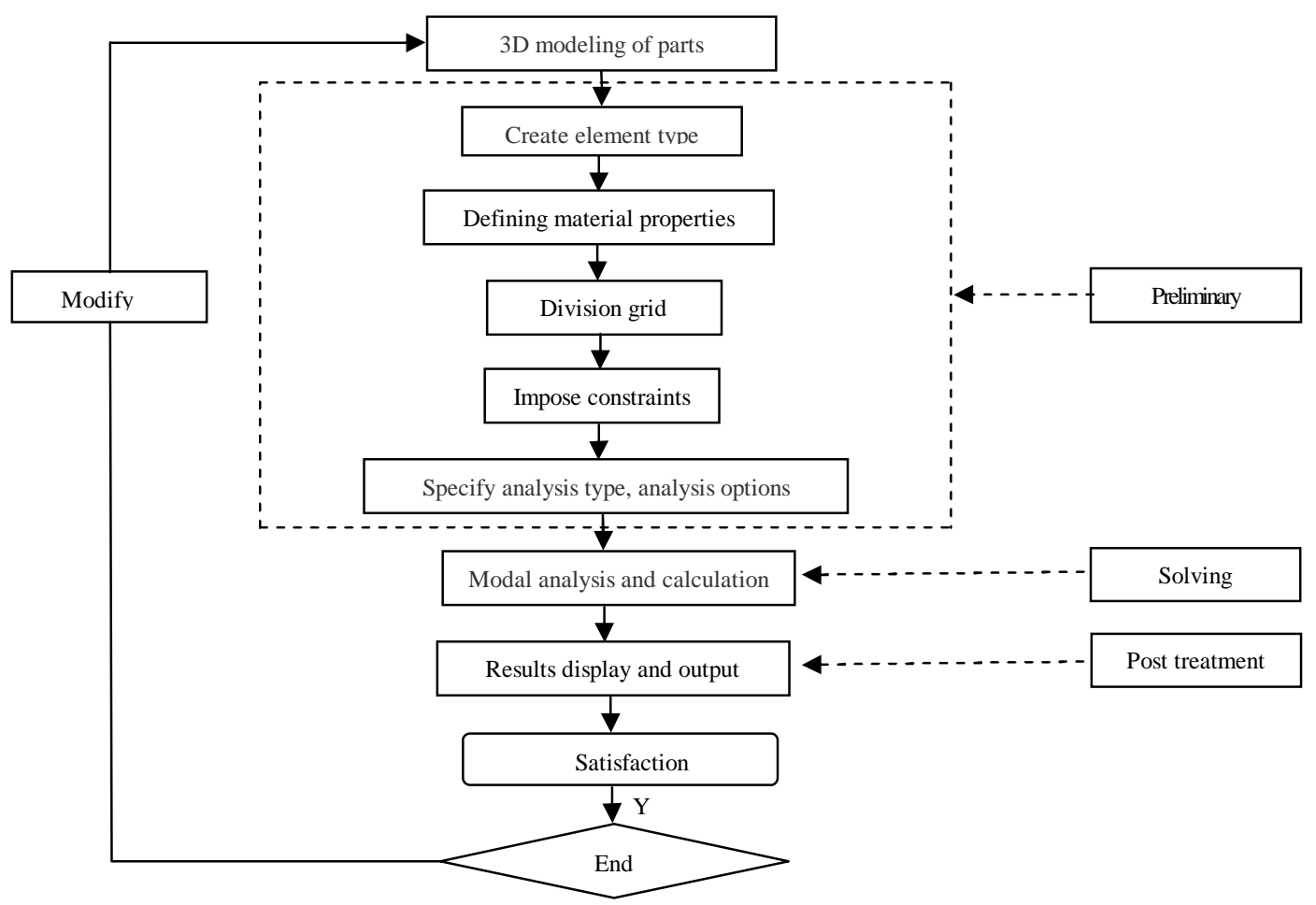

Fig. 2 Procedure of model analysis

Table.1 Six natural frequency of drag-bar

\begin{tabular}{l|lcrrrr}
\hline Exponent number & 1 & 2 & 3 & 4 & 5 & 6 \\
\hline Frequency $(\mathrm{Hz})$ & 0.237 & 0.252 & 0.319 & 0.551 & 0.739 & 0.806 \\
\hline
\end{tabular}

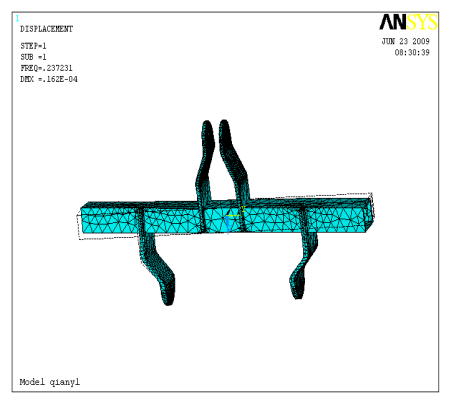

(a) First natural frequency mode

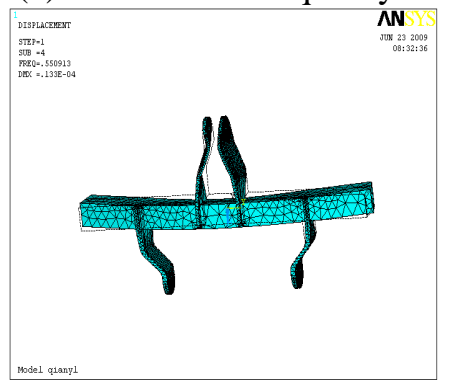

(d) Fourth

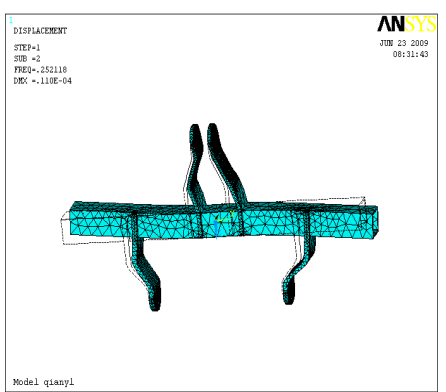

(b) Second

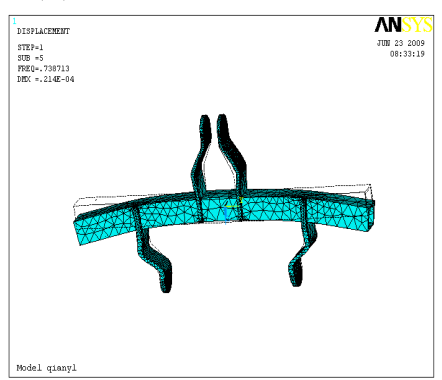

(e) Fifth

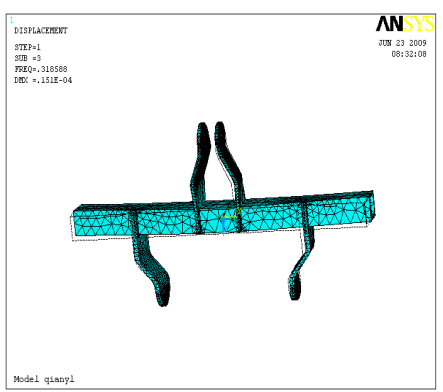

(c) Third

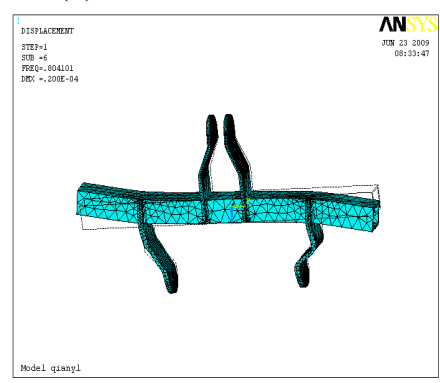

(f) Sixth

Fig.3 Natural frequency vibration model of drag-bar

Result analysis. 1) Determining modal frequency is the most basic purpose of modal analysis, because the modal frequency of the system can be determined by the frequency range of the system. From table 1 , it can be seen that in the $0 \sim 1 \mathrm{~Hz}$, the modal frequency of the drag-bar is dense, and it is the main area of the traction beam. And when the external excitation frequency and the natural frequency of the 
drag-bar are consistent, the vibration amplitude of the drag-bar is increased and the quality of the seeding is decreased. Because the working frequency of the seeding machine is generally between 6 and $7 \mathrm{~Hz}$. Therefore, the drag-bar is avoided by the operating frequency of the seeding machine, and avoids the resonance region. The drag-bar has good rigidity and reliability.

2)It can be seen from the vibration mode, the first three modes of vibration cannot see the obviously torsional deformation. To a higher order vibration mode, it will have a more significant performance. After the fourth stage mode, the radial extrusion vibration mode is appeared, which is accompanied by the torsional deformation.In the case of the first order mode, the drag-bar will be put in place as shown in Fig 3 (a) when the traction beam is vibrating at a frequency of $0.237 \mathrm{~Hz}$.

\section{Conclusions}

The 3D solid model of the planter drag-bar is modeled by Pro/E software, and the model is imported into the ANSYS software, and the finite element model of the drag-bar is established.

According to the actual conditions of drag-bar and the finite element analysis, the finite element analysis is carried out in ANSYS to get the natural frequency and vibration mode of the tractor beam. According to the analysis results, the structural strength and stiffness analysis of the drag-bar are very important for improving and enhancing the design of the drag-bar. Modal analysis method can shorten the design period, reduce the design cost and improve the design quality.

\section{Acknowledgments}

This work was financially supported by Engineering University of Armed Police Force basic research fund (WJY201307), Department of Communication Engineering background research fund(XJY201405).

\section{References}

[1]Nan Li, JOURNAL OF ANHUI AGRICULTURAL SCIENCES, J. 38(2010)19023-19024.(In Chinese)

[2]Shi-qiang QIN, Hong-ye GOU, Hong-yum JIA, Qian-hui PU, Journal of Vibration and Shock,J.34(2015)193-197.(In Chinese)

[3]D.Karayl, Z B.Barat, Biosystems Engineering, J.87(2004)437-444.

[4]Yang XU, Ren-bo SONG, Yi-su JIA,Journal of Plasticity Engineering,J.21(2014)135-141.(In Chinese)

[5]Surendra Singh, S.N.Sharma, Rajendra Prasad,Soil\&Tillage Research,J.61(2001):125-131.

[6]Wei ZHANG, Xiao-Juan ZHAO, Min LI, Development \& Innovation of Machinery \& Electrical Products,J. 28(2015)70-71.(In Chinese)

[7]Jia-sheng WANG, Shu-qi SHANG, Transactions of the Chinese Society of Agricultural Engineering,J.28(2012)65-71.(In Chinese) 